\title{
Câncer Colorretal: Características Clínicas e Anatomopatológicas em Pacientes com Idade Inferior a 40 Anos
}

\author{
Colorectal Cancer: Clinical And Anatomopathological Features \\ in Patients Below 40 Years of Age
}

\author{
JOAQUIM DAVID CARNEIRO NETO ${ }^{1}$; JOÃO BATISTAPINHEIRO BARRETO²; NATÁLIA SOUSAFREITAS ${ }^{1}$; \\ MARCELOARAÚJOQUEIROZ ${ }^{1}$ \\ ${ }^{1}$ Universidade Federal do Maranhão; ${ }^{2}$ Chefe do Serviço de Coloproctologia do Hospital \\ Universitário Presidente Dutra - HUUFMA. Hospital Universitário Presidente Dutra.
}

CARNEIRO NETO JD; BARRETO JBP; FREITAS NS; QUEIROZ MA. Câncer Colorretal: Características Clínicas e Anatomopatológicas em Pacientes com Idade Inferior a 40 Anos. Rev bras Coloproct, 2006;26(4): 430-435.

RESUMO: O câncer colorretal (CCR) é a quarta neoplasia maligna mais incidente no Brasil. Seu diagnóstico em pacientes jovens é geralmente subestimado pelos médicos por ser frequentemente considerada como condição clínica de pacientes idosos. O presente trabalho tem como objetivo avaliar variáveis clínico-patológicas em pacientes com menos de 40 anos, no que diz respeito a idade, sexo e raça do paciente, história familiar, tabagismo, sinais e sintomas, tempo entre início dos sintomas e o diagnóstico, e localização da lesão primária, através de estudo retrospectivo de 11 casos de CCR atendidos no Hospital Universitário - HUUFMA no período de 1995 a 2005 . Os pacientes incluídos representaram $11(3,27 \%)$ dos casos, sendo mais freqüente o sexo feminino (54,5\%). A idade média ao diagnóstico foi de 30,5 anos. Os sinais e sintomas mais prevalentes foram dor abdominal, alteração do hábito intestinal, perda ponderal, dor retal e hematoquezia. O tempo médio entre o início da sintomatologia e o aparecimento dos sintomas foi de 9,09 meses. A maioria das lesões encontrava-se no retossigmóide e reto (81,8\%). Cerca de $80 \%$ dos pacientes apresentaram carcinoma estágios C e D da classificação de Dukes Astler-Coller. Pacientes jovens portadores de CCR apresentam, geralmente, sintomatologia rica, com doença avançada ao diagnóstico, portanto, com menor possibilidade de cura e prognóstico mais reservado.

Descritores: Câncer colorretal; pacientes jovens; adenocarcinoma; patologia; neoplasia.

\section{INTRODUÇÃO}

O câncer colorretal (CCR) é a quarta neoplasia maligna mais incidente no Brasil ${ }^{(1)}$. Os sintomas mais prevalentes são alteração do hábito intestinal e emagrecimento, estando ambos presentes em cerca de $75 \%$ dos casos; seguidos de dor abdominal $(62,5 \%)$, hematoquezia e anemia $(37,5 \%)^{(2)}$.

O tempo médio entre o início dos sintomas e o diagnóstico varia de 2,3 meses a 10 meses. Pelo fato de os indivíduos mais jovens julgarem-se portadores de enfermidades de pequena relevância clínica, o período entre o início dos sintomas e o diagnóstico pode ser estendido ${ }^{(3)}$.
Além disso, parece haver uma tendência de realizar-se diagnóstico clínico inicial de doença benigna em pacientes jovens, sobretudo abaixo de 30 anos de idade, uma vez que a presença de neoplasia é frequentemente considerada como uma condição clínica de pacientes idosos ${ }^{(2)}$.

Em um estudo retrospectivo de 186 pacientes, idade inferior a 40 anos com adenocarcinoma colorretal primário, indicadores de maior agressividade foram encontrados em maior proporção em comparação aos pacientes com idade acima de 40 anos $^{4}$. Isto poderia explicar a alta taxa de doença avançada ao diagnóstico em pacientes com idade abaixo de 40 anos ${ }^{(5,6)}$.

Em pacientes jovens, ressalta-se a necessidade de pesquisar condições que possam estar associa-

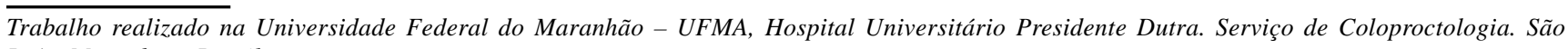
Luís, Maranhão, Brasil.

$\overline{\text { Recebido em 12/06/2006 }}$

Aceito para publicação em 26/07/2006 
das ao desenvolvimento do CCR, como as doenças genéticas (polipose adenomatosa familiar, HNPCC, síndrome do câncer colo-retal familial, síndrome de Peutz-Jeghers) ou inflamatórias e outras ${ }^{(7)}$.

\section{MATERIAL E MÉTODOS}

Realizou-se um estudo observacional transversal retrospectivo, através de dados secundários dos prontuários de pacientes submetidos à cirurgia para tratamento de câncer colorretal no Hospital Universitário Presidente Dutra - UFMA no período de março de 1995 a setembro de 2005.

Os dados foram coletados no Serviço de Assistência Médica e Estatística (SAME) do Hospital. Para isso, foi confeccionada uma ficha protocolo em que foram avaliados: idade, sexo e raça do paciente, história familiar, tabagismo, sinais e sintomas, tempo entre início dos sintomas e o diagnóstico, localização da lesão, estadiamento e estudo anatomopatológico.

$\mathrm{O}$ projeto do presente estudo foi submetido à apreciação da Comissão de Ética em Pesquisa do Hospital Universitário Presidente Dutra que deu parecer favorável à sua realização. Os dados coletados foram submetidos à análise estatística, usando-se o programa Stata SE 8.0.

Foram identificados onze pacientes com diagnóstico histopatológico de adenocarcinoma de cólon ou reto com idade inferior a 40 anos.

\section{RESULTADOS}

Dos pacientes avaliados, onze $(3,27 \%)$ foram incluídos neste estudo, dos quais seis $(54,5 \%)$ pacientes eram do sexo feminino e $5(45,5 \%)$ do sexo masculino (Figura 1). A idade média ao diagnóstico foi $30,5 \pm 5,1$, com variação de 22 a 39 anos. Dividindose a idade a cada meia década, encontrou-se maior prevalência na faixa etária compreendida entre $31 \mathrm{e}$ 35 anos, com 5 (45,5\%) dos pacientes neste grupo, seguidos de 3 pacientes $(27,3 \%)$ compreendidos na faixa etária entre 26 a 30 anos, $2(18,2 \%)$ na faixa etária entre 20 a 25 anos e somente 1 paciente $(9,0 \%)$ na faixa etária entre 36 a 40anos (Figura 2). Quanto à raça, $9,1 \%$ eram negros, $18,2 \%$ brancos e $72,7 \%$ pardos (Figura 3).

Os sinais e sintomas mais prevalentes foram: dor abdominal, presente em $72,7 \%$ dos casos; alteração do hábito intestinal que se apresentava em $63,6 \%$

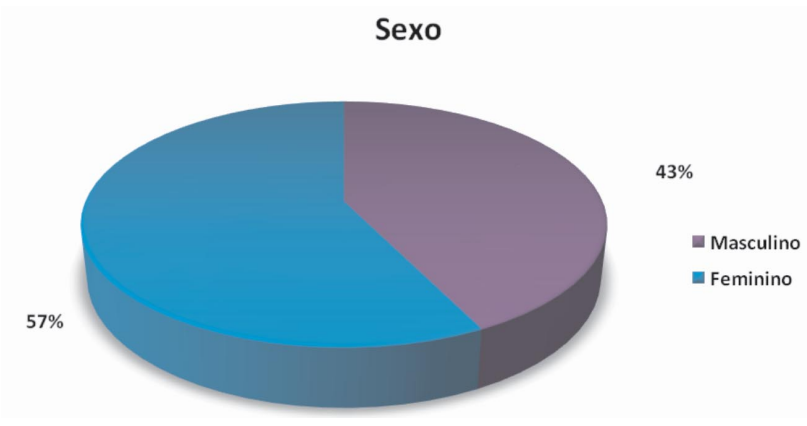

Figura 1 - Distribuição por sexo dos pacientes estudados.

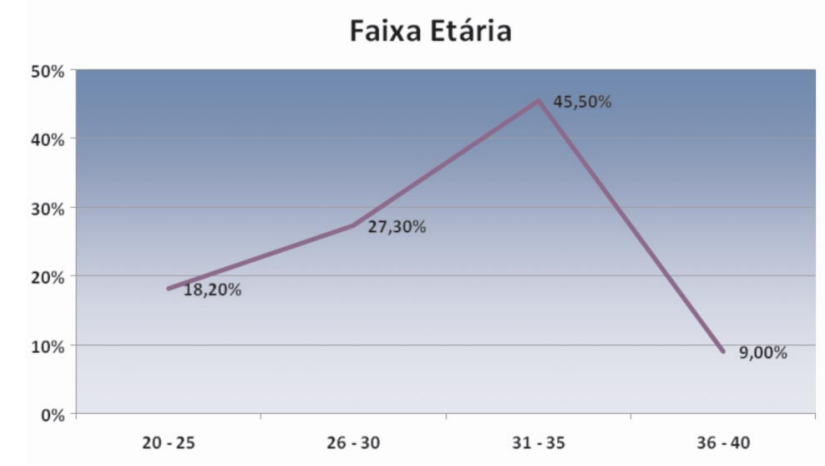

Figura 2 - Distribuição segundo a idade estratificada por meia década.

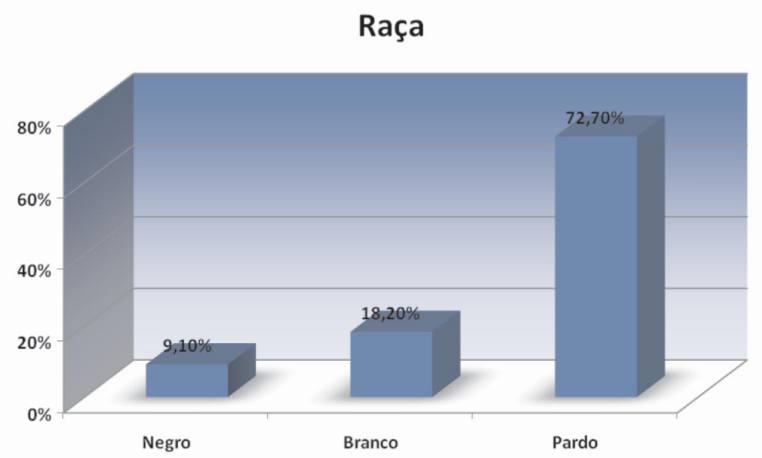

Figura 3 - Distribuição segundo a raça dos pacientes.

dos casos, além de perda ponderal, dor retal e hematoquezia, presentes em 54,5\% dos casos (Tabela 1).

O tempo médio entre o início da sintomatologia e o diagnóstico foi de 9,1 $\pm 3,6$ meses. Não foi detectada a presença de polipose colônica prévia nem polipose adenomatosa familiar em nenhum dos pacientes estudados. Devido à ausência de dados especializados nos prontuários dos pacientes, não foi possível determinar neles a presença de HNPCC, embora tenha sido possível colher a história familiar de câncer colorretal, sendo encontrada em três $(27,3 \%)$ dos pacientes estuda- 
Tabela 1 - Manifestações clínicas do câncer colorretal em pacientes com idade inferior a 40 anos.

\begin{tabular}{lc}
\hline Sintomas presentes & $\begin{array}{c}\mathbf{N}^{\mathbf{0}} \text { de pacientes }(\%) \\
(\mathbf{n}=\mathbf{1 1})\end{array}$ \\
\hline Dor abdominal & $8(72,7 \%)$ \\
Alteração do hábito intestinal & $7(63,6 \%)$ \\
Perda ponderal & $6(54,5 \%)$ \\
Dor retal & $6(54,5 \%)$ \\
Hematoquezia & $6(54,5 \%)$ \\
Muco nas fezes & $5(45,5 \%)$ \\
Tumor abdominal palpável & $3(27,3 \%)$ \\
Melena & 1 \\
Obstrução & $0(9,1 \%)$ \\
Febre & 0 \\
Outros & $5(4 \%)$ \\
\hline
\end{tabular}

dos. Em um paciente não foi possível colher dados da história familiar por desconhecimento de membros de sua família (Figura 4). Três (27,3\%) dos pacientes eram tabagistas. Não foi possível colher dados da história de tabagismo de dois pacientes (Figura 5).

Com relação à localização do tumor primário, houve predomínio do reto $(n=7 ; 63,6 \%)$, seguindo-se de retossigmóide $(\mathrm{n}=2 ; 18,2 \%)$ e cólons ascendente e transverso, presentes em iguais proporções $(n=1 ; 9,1 \%)$. Ao agrupar os tumores em relação aos cólons direito e esquerdo, notou-se prevalência deste último com 9 $(81,8 \%$ ) dos casos diagnosticados (Figura 6).

Cerca de um terço $(27,27 \%)$ dos pacientes apresentava elevação dos níveis séricos de CEA (CEA $>5 \mathrm{ng} / \mathrm{ml})$, por ocasião do diagnóstico. Em três $(27,27 \%)$ dos pacientes não foi possível obter o valor do CEA sérico.

Os tumores apresentaram tamanho médio de $54( \pm 23,8)$ x $43( \pm 21,6) \mathrm{mm}$. No entanto, em 6 casos não foi possível coletar as dimensões do tumor.

Em todos os casos estudados, os tumores corresponderam a adenocarcinoma, com moderadamente diferenciados em 7 casos $(63,6 \%)$ e pouco diferenciado em 1 caso $(9,1 \%)$. Adenocarcinomas bem diferenciados não foram encontrados nesta série. Em 3 casos $(27,3 \%)$ não foi possível coletar o tipo histológico (Figura 7).

Quanto ao comportamento biológico do CCR, observou-se invasão da serosa do órgão pelo tumor (pT3) em 4 casos $(36,4 \%)$, sendo que em 3 casos

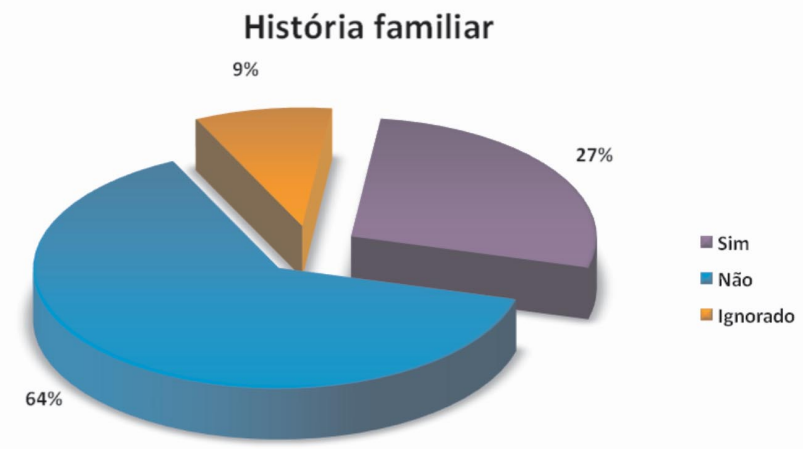

Figura 4 - História familiar de câncer colorretal.

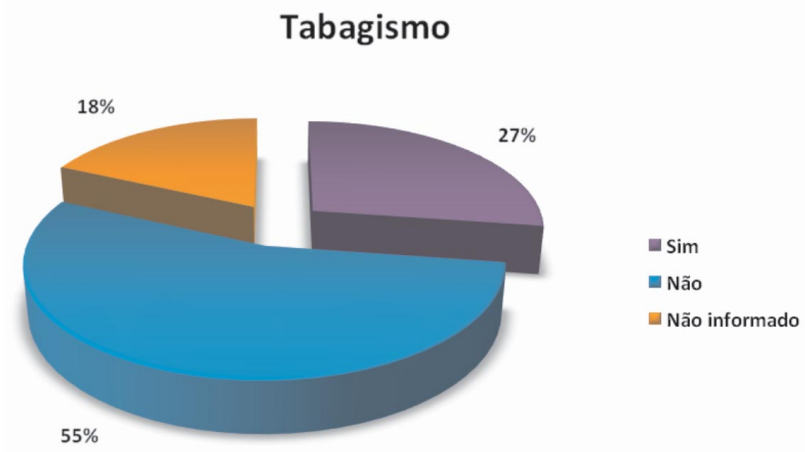

Figura 5 - Presença de tabagismo nos pacientes estudados.

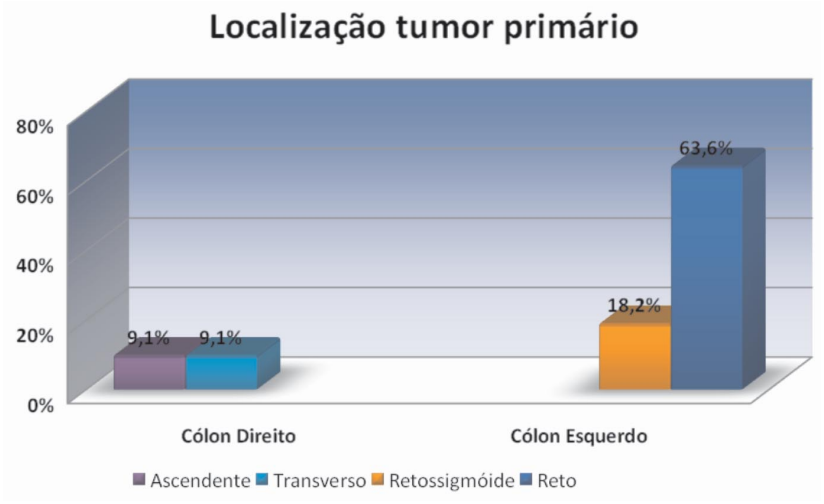

Figura 6 - Distribuição quanto à localização do tumor primário.

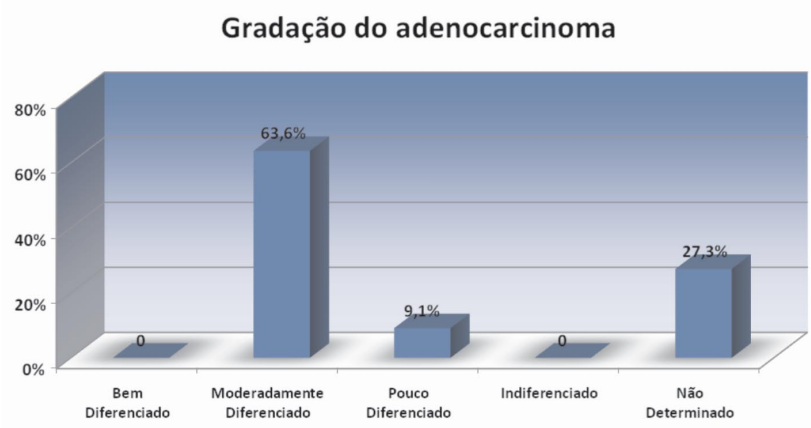

Figura 7 - Distribuição segundo a gradação do adenocarcinoma nos pacientes estudados. 
(27,3\%) havia invasão de outros órgãos ou estruturas (pT4) (Figura 8). Havia acometimento de linfonodos em seis casos $(54,6 \%)$, sendo destes, a maioria $(n=3$; $27,3 \%$ ) com quatro ou mais linfonodos acometidos (pN2). Quatro $(36,4 \%)$ dos pacientes foram classificados como pNx por serem dissecados menos de 12 linfonodos (Figura 9). Em 5 casos $(45,45 \%)$ encontrouse presença de metástase à distância.

De acordo com a classificação proposta pela União Internacional de Combate ao Câncer (UICC), encontramos: 5 pacientes $(50 \%)$ estádio IV; 3 pacientes $(30 \%)$ estádio III; e 2 pacientes $(20 \%)$ estádio I e estádio II divididos em igual proporção (Figura 10).

Segundo a classificação de Dukes Astler Coller, foram encontrados cinco pacientes $(50 \%)$ em estágio $\mathrm{D}$, dois pacientes $(20 \%)$ estágio $\mathrm{C} 2$ e um paciente nos estágios B1, B2 e C1, respectivamente. Não houve casos classificados como estágio A de Dukes AstlerColler (Figura 11).

\section{DISCUSSÃO}

A análise dos pacientes estudados mostrou discreta prevalência do sexo feminino, perfil concordante com os dados disponibilizados pelo Instituto Nacional do Câncer - INCA ${ }^{(1)}$, sendo escassa a literatura nacional para comparação.

O tempo médio decorrido entre o início da sintomatologia e o diagnóstico na série estudada foi de 9 meses, sendo este período maior que em outras séries com CCR em jovens, publicadas fora do Brasil, que foi de 6,5 meses em um estudo realizado por Bielecki et al com 15 pacientes portadores de CCR com idade inferior a 40anos ${ }^{(8)}$. Em outro estudo, foi observado tempo médio decorrido entre os primeiros sintomas e o diagnóstico de 4,9 meses ${ }^{(9)}$. Marble et al observaram que pacientes mais jovens esperam significativamente mais tempo antes de procurar a assistência médica, quando comparados aos pacientes mais velhos com idade superior a 40 anos ${ }^{(3)}$.

Em sua série de estudo nacional, Drumond et al, estudando retrospectivamente 8 pacientes portadores de CCR com idade inferior a 30 anos, encontraram uma média de 10 meses (2), valor semelhante ao encontrado no presente estudo. Provavelmente, este período mais longo, encontrado nestas séries nacionais, deva-se à maior dificuldade do acesso ao serviço de saúde em nosso país.

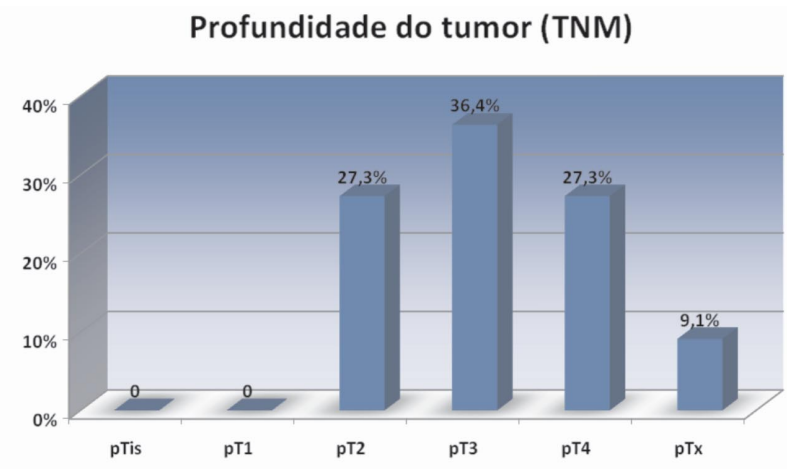

Figura 8 - Distribuição dos 11 casos de adenocarcinoma colorretal em relação à profundidade do tumor (pT) segundo o sistema TNM.

Metástase linfonodal (TNM)

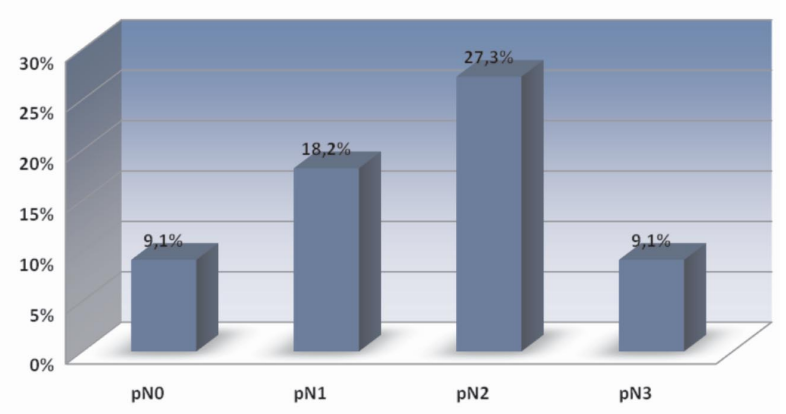

Figura 9 - Classificação da presença de metástase linfonodal (pN) segundo o sistema TNM em 11 casos de adenocarcinoma colorretal.

\section{Estadiamento TNM}

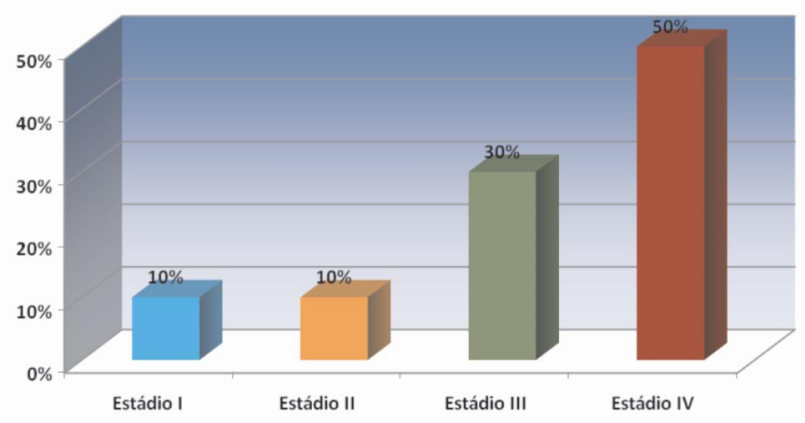

Figura 10 - Distribuição dos pacientes conforme o estadiamento TNM - AJCC/UICC.

Classificação Dukes Astler-Coller

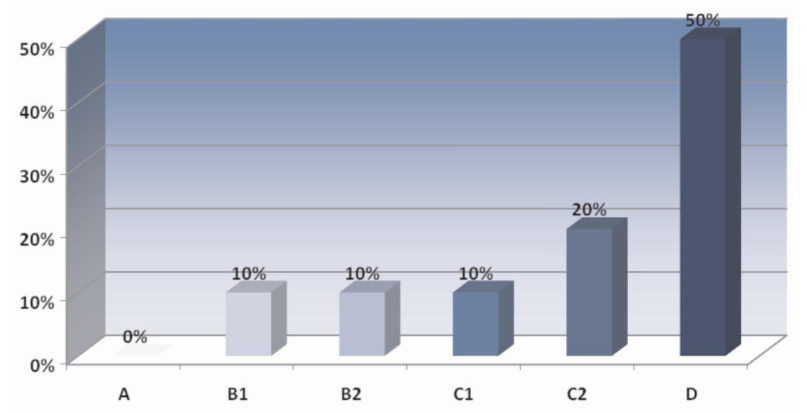

Figura 11 - Distribuição segundo a classificação de Dukes Astler-Coller. 
A associação com história familiar prévia de câncer colorretal esteve presente em $18 \%$ dos pacientes, valores superiores aos encontrados na literatura quando se diz respeito a câncer colorretal na população em geral ${ }^{(2)}$. Isto mostra a relevância da influência da história familiar como fator de risco mais importante para o CCR nesse grupo de pacientes jovens ${ }^{(10)}$. Quanto à associação com tabagismo, aproximadamente um terço $(27 \%)$ apresentava história, reforçando assim a necessidade de uma maior intensificação de campanhas de prevenção.

No presente estudo, houve um predomínio de tumores no cólon esquerdo, principalmente sigmóide e reto, semelhante ao encontrado na literatura ${ }^{(8,11)}$. Estudos recentes revelam tendência a um aumento da incidência de tumores no cólon direito (12). Alguns autores tentam justificar esta distribuição topográfica pelo fato de o cólon ter características embriológicas e biológicas diferentes, podendo estar envolvidas na patogênese do CCR por mecanismos distintos e, consequentemente, comportamento biológico diferente ${ }^{(12)}$.

Cerca de um terço dos pacientes apresentava elevação dos níveis séricos de CEA, por ocasião do diagnóstico. Estes valores vêm corroborar as afirmações de que este antígeno não tem valor diagnóstico, sendo útil apenas para prognóstico e acompanhamento.

O CCR em pacientes jovens (até 40 anos) foi descrito como mais volumoso, mais avançado e com indicadores histopatológicos de maior grau de agressividade, como pouca diferenciação, invasão de vasos linfáticos, sanguíneos e nervos, além de uma maior incidência de tumores mucossecretores ${ }^{(11)}$. Na presente série, o tamanho médio dos tumores primários na ocasião do diagnóstico, com médias de $54( \pm 23,8)$ x 43 $( \pm 21,6) \mathrm{mm}$ foi considerado grande, considerando-se parâmetros utilizados por Farhoud et al em um estudo com 320 pacientes de câncer colorretal submetidos a tratamento cirúrgico no Serviço de Gastroenterologia Cirúrgica do Hospital do Servidor Público Estadual entre 1964 e 1980, quando tumores pequenos mediam em seu maior diâmetro $35 \mathrm{~mm}$ ou menos, e grandes com diâmetro acima de $35 \mathrm{~mm}^{(13)}$.

O estadiamento anatomopatológico apresentase como a principal ferramenta para, ao ser utilizada isoladamente, podermos inferir sobre o prognóstico de doentes portadores de CCR ${ }^{(7,14)}$, com a grande maioria dos trabalhos publicados até a atualidade que ressaltam a maior frequiência de tumores em estágio avan- çado (Dukes C/D ou TNM III/IV) entre os pacientes jovens, sendo em algumas séries a única variável prognostica independente ${ }^{(7)}$.

A maioria dos nossos pacientes apresentou estadiamento avançado, tanto pela classificação de Dukes como pelo sistema TNM, com invasão da serosa (pT3 e pT4) observada em sete casos $(63,7 \%)$, e em seis casos $(54,6 \%)$ observou-se a presença de células metastáticas nos linfonodos do mesocólon. Foram identificadas metástases à distância em cinco casos $(45,5 \%)$, o que reflete retardo do diagnóstico correto na maioria dos pacientes que, por isto, apresentaram neoplasias mais avançadas, comprometendo assim o prognóstico. Isto pode ser explicado pelo fato de nossos pacientes pertencerem a camadas sócio-econômicas menos favorecidas e que têm, portanto, menor acesso aos serviços de saúde. Além disto, há subestimação por parte do médico assistente por julgar erroneamente a sintomatologia do paciente portador de CCR com idade inferior a 40 anos como doença benigna pelo fato de o paciente ser jovem. Desta forma, se faz necessária uma melhor formação acadêmica dos médicos no sentido de reforçar a importância do diagnóstico precoce do câncer colorretal.

Em países desenvolvidos, como os Estados Unidos, onde se tem maior preocupação com o diagnóstico precoce e campanhas de prevenção e conscientização mais intensas da população, observase fenômeno contrário, com a maioria dos pacientes sendo diagnosticada em estágios mais iniciais da doença ${ }^{(15)}$.

\section{CONCLUSÃO}

Pacientes com idade inferior a 40 anos apresentam geralmente sintomatologia rica, geralmente com doença avançada ao diagnóstico, diminuindo a possibilidade de cura e, portanto, com prognóstico mais reservado. Isto ocorre, provavelmente, pela maior tendência de os pacientes mais jovens retardarem sua procura à assistência médica e pelo fato de o diagnóstico de câncer colorretal nestes pacientes ser frequentemente ignorado, ao ser muitas vezes considerado como uma condição clínica de pacientes idosos. Reforçando a necessidade do diagnóstico precoce, campanhas educativas de prevenção e uma melhor formação dos médicos, que devem ficar atentos à possibilidade de CCR, mesmo em pacientes jovens, ao detectarem sinais e sintomas sugestivos desta patologia. 


\begin{abstract}
The colorectal cancer (CRC) is the fourth incident malignant neoplasm in Brazil. Its diagnosis in young patients is usually underestimated by being frequently considered as elderly patients' clinical condition. This research has the objective to evaluate clinical-pathological variables in patients below 40 years, according to age, gender, ethnic, family history, smoking, signs and symptoms, time from the beginning of the symptoms to the diagnosis, and location of the primary lesion through retrospective study of 11 cases of CRC assisted at the Academical Hospital - HUUFMA in the period from 1995 to 2005 . The included patients represented $11(3,27 \%)$ of the cases which are more frequent in women $(54,5 \%)$. The average age to the diagnosis was 30,5 years. The signs and symptoms more prevalent were abdominal pain, intestinal habit alterations, weight loss, rectal pain and hematoquezy. The average time from the beginning of the sintomatology to the emergence of the symptoms was of 9,09 months. Most of the lesions were founded in the recto-sigmoid and rectum $(81,8 \%)$. Around $80 \%$ of the patients presented carcinoma in stages C and D of Dukes Astler-Coller's classification. Young patients with CRC have usually vast symptomatology with advanced disease at the diagnosis, therefore with smaller cure possibility and worse prognosis.
\end{abstract}

Key-words: Colorectal cancer; young patients; adenocarcinoma; pathology; neoplasm.

\section{REFERÊNCIAS}

1. BRASIL. Ministério da Saúde. Instituto Nacional de Câncer. Estimativa 2005: Incidência de câncer no Brasil. Rio de Janeiro: INCA 2004. 94p. il.

2. $\quad$ DRUMOND, C.A.; FERRO, R.A.F.; NOGUEIRA, A.M.F.; PROFETA da LUZ, M.M.; CONCEIÇÃO, S.A.; SILVA, R.G.; LACERDA FILHO, A. Câncer colorretal em pacientes com idade inferior a 30 anos. Rev Bras Coloproct jul./set. 2003; 23 (3): p 147-154.

3. MARBLE, K.; BANERGEE, S.; GREENWALD, L. Colorectal carcinoma in young patients. J Surg Oncol 1992; 51: p 179-182.

4. CUSACK, J.C.; GIACCO, G.G.; CLEARY, K. DAVIDSON, B.S.; IZZO, F.; SKIBBER, J.; YEN, J.; CURLEY, S.A. Survival factors in 186 patients younger than 40 years old with colorectal adenocarcinoma. J Am Coll Surg 1996; 183: p 105112.

5. LEE, P.Y.; FLETCHER, W.S.; SULLIVAN, E.S.; VETTO, J.T. Colorectal cancer in young patients: characteristics and outcome. Am Surg 1994; 60: p 607-612.

6. MINARDI JR., A.J.; SITTING, K.M.; ZIBARI, G.B.; McDONALD, J.C. Colorectal cancer in the young patient. Am surg 1998; 64: p 849-853.

7. LUPINACCI R.M.; CAMPOS F.G.C.M.; ARAÚJO S.E.A.; IMPERIALE A.R; SEID V.E.; HABR-GAMAA.; KISS D.R.; GAMA-RODRIGUES, J.J. Análise comparativa das características clínicas, anátomo-patológicas e sobrevida entre pacientes com câncer colo-retal abaixo e acima de 40 anos de idade. Rev bras Coloproct jul./set. 2003; 23 (3): p 155-162.

8. BIELECKI, K.; KAMIÑSKI, P.; KLUKOWSKI, M.; WLODARCZYK, A. Colorectal carcinoma in patients under 40 years of age: clinical presentation and early effects of treatment. Mon Sci Monit 1997 ; 3 (4) : p 499-503.
9. MACGILLIVRAY, D.C.; SWARTZ, S.E.; ROBINSON, A.M.; CRUESS, D.F.; SMITH L.E. Adenocarcinoma of the colon and rectum in patients less than 40 years of age. Surg Gynecol Obstet 1991; 172: p 01-07.

10. TURKIEWIEZ, D.; MILLER, B.; SCHACHE, D.; COHEN, J.; THEILE, D. Young patients with colorectal cancer: How do they fare? ANZ J Surg 2001; 45: p 209-212.

11. MENDES, M.B.P; COSTA, L.D.B.; TAMURA, S. Tumores do cólon e reto: experiência de 16 anos. Rev Bras Coloproct 1992; 12 (4): p 132-138.

12. GERVAZ, P. et al. Dukes B colorectal cancer: distinct categories and clinical outcome based on proximal or distal tumor location. Dis Colon Rectum 2001. 44 (3): p 364-373.

13. FARHOUD, S.; BROMBERG, S.H.; BARRETO, E. et al. Clinical and macroscopic variables of colorectal carcinoma that influence the prognosis. Arq. Gastroenterol jul./set. 2002; 39 (3): p 163-172.

14. BÜLOW S. Colorectal cancer in patients less than 40 years of age in Denmark, 1943-1967. Dis Colon Rectum 1980; 23: p 327-336.

15. CHEN, L.K.; HWANG, S.J.; LI, A.F.Y.; LIN, J.K.; WU, T.C. Colorectal cancer in patients 20 year old or less in Taiwan. Southern Med J dez. 2001.94 (12): p 1202-1205.

Endereço para Correspondência: JOÃO BATISTA PINHEIRO BARRETO CPM - Clínica de Proctologia do Maranhão Renascença Medical Center

Avenida Collares Moreira, 11 - Quadra 23 - 5 Andar Renascença II

Fone/Fax: (98) 3227-4332

E-mail:jbpbarreto@uol.com.br / jdavidneto@hotmail.com 